\title{
Visualization of Nigrosome 1 from the Viewpoint of Anatomic Structure
}

\author{
(D) N. Arai, (D). Kan, (D). Ogawa, (D). Uchida, (D). Takizawa, (D). Omori, (D)T. Miyati, (D). Kasai, (D). Kunitomo, and \\ (iD) Y. Shibamoto
}

\section{ABSTRACT}

BACKGROUND AND PURPOSE: Parkinson disease is related to neurodegeneration and iron deposition in the substantia nigra pars compacta and nigrosome 1. However, visualization of nigrosome 1 via MR imaging is poor owing to the bilateral asymmetry, regardless of whether it is healthy. We focused on the magic angle and susceptibility effect and evaluated the anatomic slant structure of nigrosome 1 by tilting subjects' heads in the $\mathrm{B}_{0}$ direction.

MATERIALS AND METHODS: To investigate the effectiveness of the magic angle, we tilted the volunteers' heads to the right and left in the $B_{0}$ direction or not at all for evaluating correlations between the degree of head tilting and visualization of the right nigrosome 1 and left nigrosome 1 using 3D spoiled gradient-echo sequences with multiecho acquisitions. We evaluated the susceptibility of nigrosome 1 and the local field using quantitative susceptibility mapping to assess static magnetic field inhomogeneity.

RESULTS: The heads tilted to the right and left showed significantly higher contrasts of nigrosome 1 and the substantia nigra pars compacta than the nontilted heads. No significant differences were observed in the visualization and susceptibility between the right nigrosome 1 and left nigrosome 1 for each head tilt. The effect of the magic angle was remarkable in the nontilted heads. This finding was supported by quantitative susceptibility mapping because the anatomic slant structure of nigrosome 1 was coherent between the axis of nigrosome 1 and the magic angle.

CONCLUSIONS: The asymmetric visualization of nigrosome 1 is affected by the magic angle and susceptibility. The anatomic slant structure of nigrosome 1 causes these challenges in visualization.

ABBREVIATIONS: $\mathrm{PD}=$ Parkinison disease; $\mathrm{SNc}=$ substantia nigra pars compacta

$\mathbf{P}$ arkinson disease (PD) is associated with major pathologic degeneration of dopaminergic neurons, mainly in the basal ganglia and especially in the substantia nigra pars compacta (SNc). ${ }^{1}$ It has been reported that PD or other neurodegenerative disorders include iron accumulation in the SNc. ${ }^{2-6}$ In addition, normally, the iron load in the basal ganglia increases with age, ${ }^{7,8}$

Received June 29, 2019; accepted after revision October 10.

From the Department of Radiology (N.A., H. Kasai, H. Kunitomo), Nagoya City University Hospital, Nagoya, Japan; Radiological and Medical Laboratory Sciences (H. Kan), Nagoya University Graduate School of Medicine, Nagoya, Japan; Departments of Radiology (H. Kan, M.O., Y.S.), and Neurology (Y.U.), Nagoya City University Graduate School of Medical Sciences, Nagoya, Japan; Healthcare Business Unit (M.T., K.O.), Hitachi Ltd, Tokyo, Japan; and Division of Health Sciences, Graduate School of Medical Science (T.M.), Kanazawa University, Kanazawa, Japan.

The sponsor of this research is Hitachi Ltd.

Paper previously presented at: Annual Meeting of the European Congress of Radiology, March 1-5, 2017; Vienna, Austria (C-1756); and Annual Meeting of the Japanese Society of Radiological Technology, April 13-16, 2017; Yokohama, Japan. Please address correspondence to Nobuyuki Arai, MS, Department of Radiology, Nagoya City University Hospital, 1-Kawasumi, Mizuho-cho Mizuho-ku, Nagoya, Aichi, 4678602, Japan; e-mail: rarai51@med.nagoya-cu.ac.jp

http://dx.doi.org/10.3174/ajnr.A6338 and the best indicators of physiologic aging are iron deposition in the putamen and microstructural damage and atrophy in the thalamus. ${ }^{1}$

Increased SNc iron content generally indicates that there is dopaminergic neuronal loss. However, the degree of dopaminergic neuronal loss in PD is higher in the nigrosomes than in the other subregions of the substantia nigra with the maximum loss occurring in nigrosome 1.9,10 Recent studies have suggested that nigrosome 1 is the largest cluster of neurons within the nigrosomes and it is highly attenuated. ${ }^{11}$ It is located in the caudal and mediolateral part of the SNc and is associated with PD.,10,12-14

From a neuroradiologic point of view, MR imaging shows nigral changes, and changes in $\mathrm{T}^{*}$ relaxation times seem to be associated with increased iron deposition in the SNc that occurs in some cases of PD. It is essential to determine which conditions affect iron deposition in nigrosome 1 because PD is closely related to neurodegeneration of the $\mathrm{SNc}$ and especially nigrosome 1. Nigrosome 1 has been evaluated by a combination of images obtained with a 3D spoiled gradient-echo technique and with a multiecho technique. ${ }^{11,15,16}$ As PD progresses, the MR imaging 
signals of nigrosome 1 commonly diminish; this feature reflects iron accumulation and neurodegeneration. However, the MR imaging visualization of nigrosome 1 is often poor because of the asymmetry of this cell cluster, regardless of whether nigrosome 1 is healthy. In addition, little attention has been paid to poor visualization due to asymmetry. This is important because poor visualization could result in misclassification of a healthy or abnormal nigrosome 1 .

In this study, we explored the cause of nigrosome 1 being poorly visualized despite healthy conditions. We focused on the magic angle and the susceptibility effect, and we evaluated the anatomic slant structure (head tilt) of nigrosome 1 in the $B_{0}$ direction using combined multi-gradient-echo images and by tilting the subjects' heads in the $\mathrm{B}_{0}$ direction.

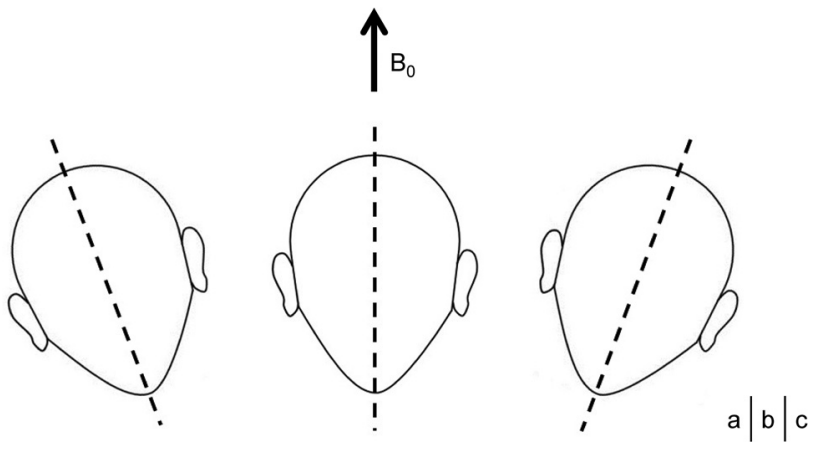

FIG 1. Volunteers' heads were held in 1 of 3 ways: tilted to the right $(A)$, not tilted $(B)$, and tilted to the left $(C)$ in the $B_{0}$ direction.

\section{MATERIALS AND METHODS \\ Subjects}

Fourteen young healthy volunteers ( 3 women and 11 men; mean age, 25 years; ranging from 22 to 31 years) participated in the study. This study was approved by the institutional review board at Nagoya City University. The volunteers participated in the study of their own free will, and informed consent was obtained from all the participants.

\section{Data Analysis}

The volunteers' heads were tilted to the right $\left(20.9^{\circ} \pm 5.8^{\circ}\right)$ or left $\left(18.9^{\circ} \pm 6.6^{\circ}\right)$ in the $\mathrm{B}_{0}$ direction or not at all, to investigate the effectiveness of the magic angle (Fig 1). The 3D spoiled gradientecho sequences with multiecho acquisitions targeted the midbrain and were oriented orthogonally across from the anterior midbrain (Fig $2 A,-B$ ). We evaluated the correlations between the degree of head tilting and visualization of the right nigrosome 1 and left nigrosome 1 . To calculate the contrast of nigrosome 1 and the remaining $\mathrm{SNc}$ that was positioned around nigrosome 1 to assess its visualization, we used the following equation:

$$
\text { Contrast }=\frac{S I_{n 1}-S I_{S \mathrm{Nc}}}{S I_{n 1}+S I_{S \mathrm{Nc}}},
$$

where $S I_{n 1}$ and $S I_{\mathrm{SNc}}$ are the signal intensities of nigrosome 1 and $\mathrm{SNc}$, respectively (Fig 2C). We calculated the SDs of the contrasts of the right nigrosome 1 and left nigrosome 1 and the $\mathrm{SNc}$ for each head tilt, to evaluate the reproducibility of contrast measurement. The contrast was measured 6 times in 1 volunteer using right head tilting and left head tilting. We used ImageJ 1.48 imageprocessing software (National Institutes of Health, Bethesda, Maryland) to analyze the images.

In addition, we evaluated the susceptibility of nigrosome 1 and the local field using quantitative susceptibility mapping ${ }^{16,17}$ to assess the visualization of nigrosome 1 changed by the phase modulation due to the dipole effect. ${ }^{18-20}$ To estimate the tissue-generated local field map and the susceptibility map, we used the multi-spoiled gradient-echo sequence in healthy volunteers. First, the local field map was estimated from the acquired multiphase images by means of sophisticated harmonic artifact reduction using the phase data method with variable kernel sizes from 0.75 at the boundary of the brain to $30 \mathrm{~mm}$ toward the

FIG 2. The 3D spoiled gradient-echo sequences with multiecho acquisitions are oriented orthogonally to the anterior midbrain $(A)$, and nigrosome 1 is visualized in the caudal and mediolateral part of the substantia nigra pars compacta $(B)$. When the subjects' heads were tilted to the right or left, the MR imaging signal intensities measured on the basis of the depicted ROI (C) differentiated nigrosome 1 and SNc.

Table 1: Number of visualizations of discriminable nigrosome 1 when the subjects' heads were tilted in the $B_{0}$ direction $(n=14)$

\begin{tabular}{|c|c|c|c|c|c|c|}
\hline \multirow{2}{*}{$\frac{\text { Tilting Head in } \mathrm{B}_{0} \text { Direction }}{\text { Nigrosome } 1}$} & \multicolumn{2}{|c|}{ Right } & \multicolumn{2}{|c|}{ None } & \multicolumn{2}{|c|}{ Left } \\
\hline & Right & Left & Right & Left & Right & Left \\
\hline Discriminable nigrosome 1 (No.) & 9 & 9 & 6 & 5 & 10 & 11 \\
\hline
\end{tabular}




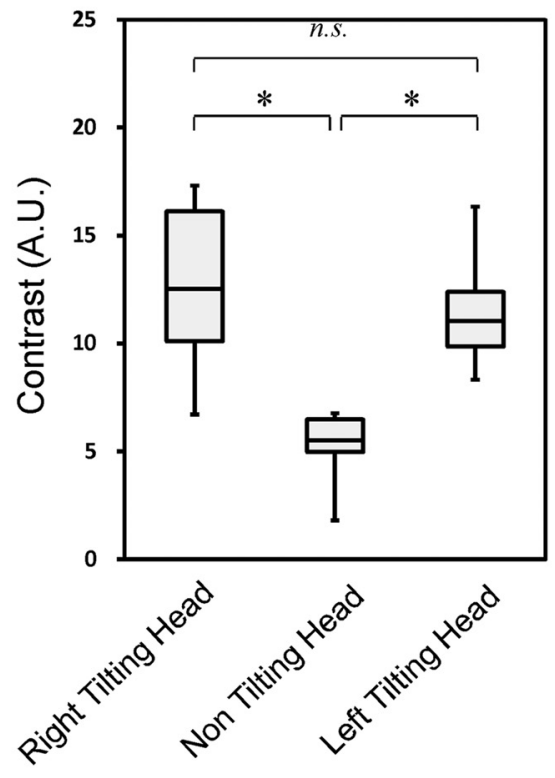

Right Nigrosome 1

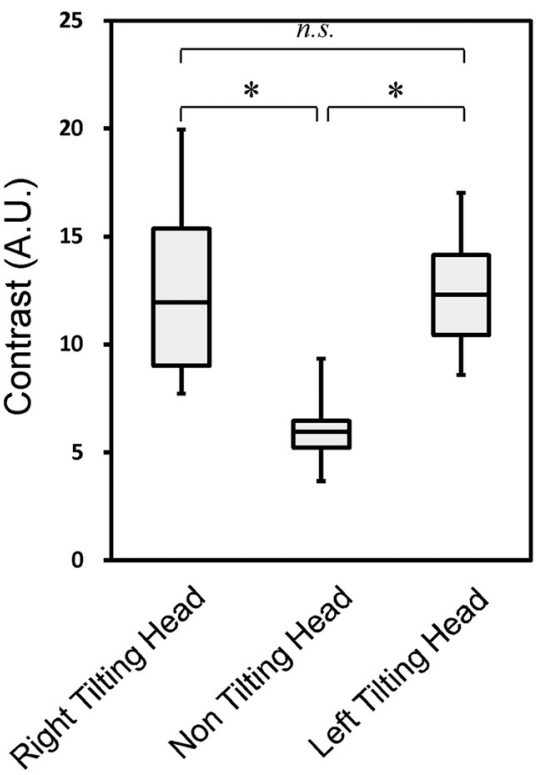

Left Nigrosome 1
* : $p<0.05$

n.s. : not significant

FIG 3. Visualization of the right $(A)$ and left $(B)$ nigrosome 1 , in which the volunteers' heads were tilted to the right or left in the $B_{0}$ direction or not at all.
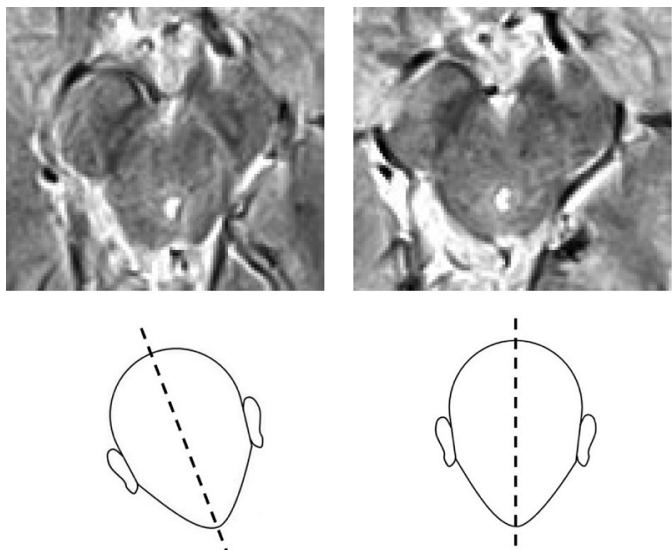
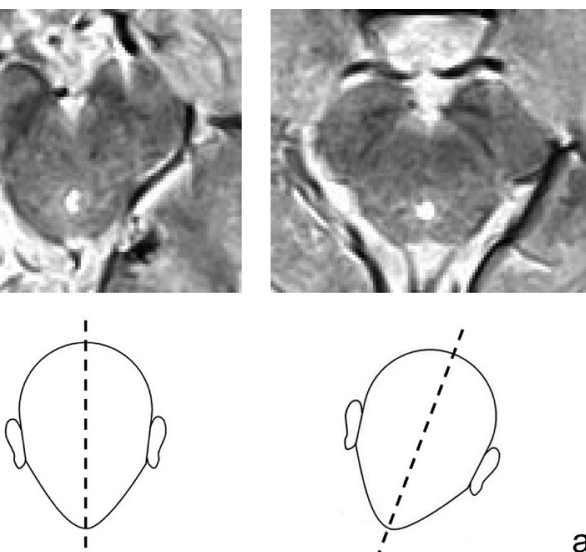

FIG 4. Examples of visualization of nigrosome 1 with a right head tilt $(A)$, without a head tilt $(B)$, and with a left head tilt $(C)$ in the $B_{0}$ direction.

center of the brain. ${ }^{21,22}$ Then, the susceptibility map was reconstructed with an improved sparse linear equation and the least-squares algorithm. ${ }^{23}$ All data were processed with the in-house Matlab R2018a (MathWorks, Natick, Massachusetts) program.

\section{MR Imaging Acquisition}

All examinations were performed on a 3T MR imaging system (Trillium Oval; Hitachi, Tokyo, Japan) with a 15- or 32-channel head matrix coil. The evaluation was based on a combination of 3D spoiled gradient-echo images with 9 echoes. To combine the 3D spoiled gradient-echo with each TE magnitude, we used the following parameters: TR, $83 \mathrm{~ms}$; minimum and maximum TE, 18.5 and $55.3 \mathrm{~ms}$, respectively (number of combined echoes, 9; echo spacing, $4.6 \mathrm{~ms}$ );

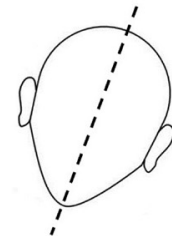

$\mathrm{a}|\mathrm{b}| \mathrm{c}$ flip angle, $10^{\circ}$; section thickness, $1.5 \mathrm{~mm}$; matrix, $300 \times 300$; FOV, $190 \mathrm{~mm}$ (in-plane resolution, 0.63 $\times 0.63 \mathrm{~mm}$ ); parallel imaging factor, 1.1; number of signal averages, 1 ; receiver bandwidth, $210 \mathrm{kHz}$; and acquisition time, 4 minutes 53 seconds. The receiver bandwidth was fixed for each TE in the multiecho imaging technique. Quantitative susceptibility mapping was performed using the following parameters: TR, $34 \mathrm{~ms}$; TE, 6-30 ms at 6-ms intervals; flip angle, $15^{\circ}$; section thickness, $1.0 \mathrm{~mm}$; matrix, $192 \times 192$; FOV, $192 \mathrm{~mm}$ (in-plane resolution, $1.0 \times 1.0 \mathrm{~mm}$ ); parallel imaging factor, 1.9; number of signal averages, 1 ; receiver bandwidth, $77 \mathrm{kHz}$; and acquisition time, 10 minutes 5 seconds.

\section{Statistical Analysis}

A Wilcoxon signed rank test with a Bonferroni correction for non-normally distributed data was performed to examine the relationship between the visualization of nigrosome 1 and the degree of head tilting to the right and left. Statistical analysis was performed using SPSS (IBM, Armonk, New York). $P<.05$ and $P<.017$ (.05/3 after Bonferroni correction based on 3 comparisons per task) were considered statistically significant.

\section{RESULTS}

The visualization of nigrosome 1 depends on the difference in the field strength; however, it should be visualized bilaterally, 
Table 2: Results of contrast measurement repeated 6 times in 1 volunteer with right head tilting and left head tilting

\begin{tabular}{|c|c|c|c|c|}
\hline \multirow{2}{*}{$\frac{\text { Tilting Head in } \mathrm{B}_{0} \text { Direction }}{\text { Nigrosome } 1 \text { and } \mathrm{SNc}}$} & \multicolumn{2}{|c|}{ Right } & \multicolumn{2}{|c|}{ Left } \\
\hline & Right & Left & Right & $\overline{\text { Left }}$ \\
\hline \multicolumn{5}{|l|}{ Times } \\
\hline 1 & 12.0 & 11.8 & 10.6 & 12.6 \\
\hline 2 & 12.1 & 11.4 & 11.0 & 12.3 \\
\hline 3 & 12.3 & 11.4 & 10.6 & 13.0 \\
\hline 4 & 11.9 & 11.9 & 10.4 & 12.4 \\
\hline 5 & 12.5 & 11.0 & 10.5 & 12.6 \\
\hline 6 & 12.3 & 11.4 & 10.9 & 12.4 \\
\hline SD & 0.20 & 0.32 & 0.21 & 0.26 \\
\hline
\end{tabular}
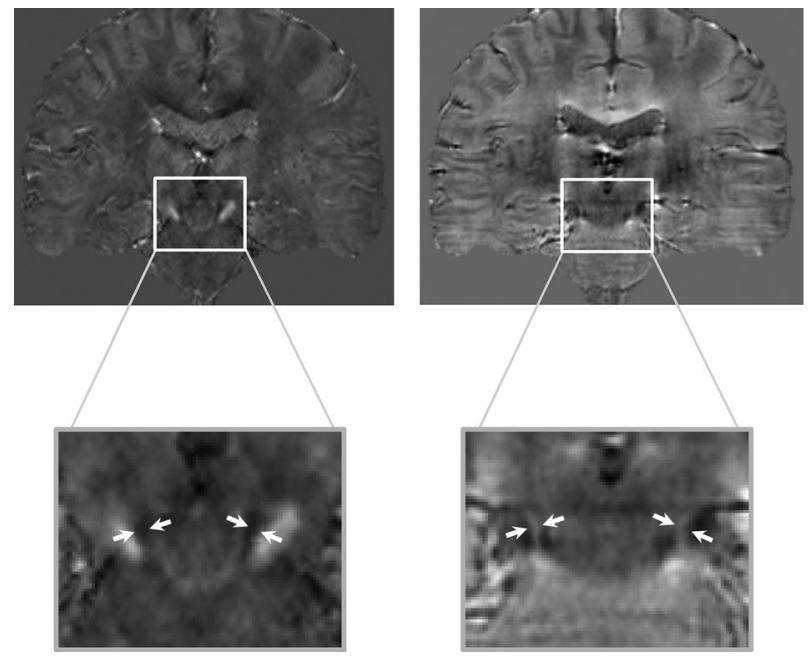

FIG 5. Coronal sections of the 3D susceptibility map $(A)$ and the local field map $(B)$. The anatomic slant structure of nigrosome 1 is coherent between the axis of nigrosome 1 (arrows) and the magic angle that occurs at approximately $54.7^{\circ}$ in the $\mathrm{B}_{0}$ direction by dipolar interaction (C).

especially when visualizing a healthy nigrosome 1 . Poor visualization due to asymmetry of the bilateral nigrosome 1 was discovered, though all the subjects in this study were young and healthy and had no nucleus degeneration. Additionally, in some subjects, we were unable to find a discriminable nigrosome 1 (Table 1).

The heads tilted to the right and left showed a significantly higher contrast of nigrosome 1 and SNc than the nontilted heads (Fig 3). Figure 4 shows examples of the visualization of nigrosome 1 with a right head tilt $(A)$, without a head tilt $(B)$, and with a left head tilt $(C)$. No significant differences were observed in the visualization and susceptibility between the right nigrosome 1 and left nigrosome 1 for each head tilt. The contrast of nigrosome 1 and SNc showed sufficient reproducibility among the 6 measurements (Table 2). However, the effect of the magic angle was remarkable in the nontilted heads and was also supported by quantitative susceptibility mapping. The boundary of nigrosome 1 and $\mathrm{SNc}$ is likely visible (Fig $5 A,-B$ ), because according to the anatomic slant structure of nigrosome 1 along the magic angle, the dipolar interaction has angular dependence (Fig 5C).

\section{DISCUSSION}

Conventional MR imaging characteristics of the substantia nigra in PD involve increased iron-related contrast enhancement, which reflects the sensitivity of the gradient-echo sequences to

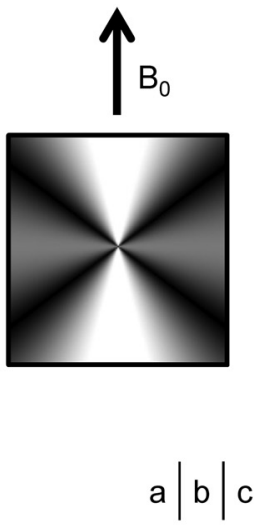

the resulting changes in $\mathrm{R}_{2}{ }^{*}$ relaxivity - that is, the signal intensity on a $3 \mathrm{D}$ spoiled gradient-echo is diminished specifically in the lateral portion of the SNc. Iron is the main source of susceptibility in the regions of the nucleus in the midbrain. In addition, a boundary is visible between nigrosome 1 and $\mathrm{SNc}$ on gradient-echo images because they naturally have different degrees of iron deposition. The important point here is that the degree of iron deposition is lower than that of neurodegeneration in the midbrain.

In principle, one would expect to visualize a healthy nigrosome 1 bilaterally on MR imaging, but the visualization is often poor because of its asymmetry. Furthermore, SNc is also asymmetric. Hence, a healthy nigrosome 1 and an abnormal nigrosome 1 might be misclassified. The problem that we have assessed here (ie, misclassification of a healthy nigrosome 1 and an abnormal nigrosome 1) has seldom been studied.

The differences in visualization were due to the way in which the subjects' heads were tilted (ie, to the right or left), which caused asymmetric visualization of nigrosome 1 . In our study, we found that when the heads were tilted to the right or left, the contrast between nigrosome 1 and $\mathrm{SNc}$ on the images was significantly higher than that with the nontilted heads. Furthermore, the results in Fig 4 strongly suggest the reproducibility of contrast measurements by manually drawing the ROIs and measuring the signal intensities of nigrosome 1 and SNc. We focused on the magic angle in the $\mathrm{B}_{0}$ direction. The magic angle that occurs at approximately $54.7^{\circ}$ in the $\mathrm{B}_{0}$ direction and so forth along the axis of the magic angle ${ }^{24,25}$ accounts for interference with this boundary in the nontilted heads. The relationship between the poor visualization of nigrosome 1 and the magic angle causes asymmetric visualization of nigrosome 1 (Table 1 and Fig 3).

We evaluated the anatomic slant structure of nigrosome 1 using quantitative susceptibility mapping, which enables quantitative investigations of the iron content in tissues. ${ }^{17,21,26-29} \mathrm{R}_{2}{ }^{*}$ increases exponentially with increasing age, ${ }^{7,8}$ but all the subjects included in this study were young and healthy without any nucleus degeneration. Hence, nigrosome 1 and the local field were 
not assessed with susceptibility-weighted imaging and the $\mathrm{T} 2^{*}$ image technique; instead, quantitative susceptibility mapping, which is more sensitive, was used. The results indicated that the anatomic slant structure of nigrosome 1 was coherent between the axis of nigrosome 1 and the magic angle (Fig 5).

Quantitative susceptibility mapping can also be used for the detection and quantification of iron deposition, whereas the status of the nuclear regions of the midbrain is assessed through diffusion tensor imaging. The bulk tissue microstructure can be measured with this technique on the basis of the tissue microenvironment with mean diffusivity and fractional anisotropy. The mean diffusivity increases with microscopic barrier disruption and extracellular fluid accumulation, and fractional anisotropy provides information on the microstructural integrity of highly oriented microstructures (eg, myelin). ${ }^{30,31}$ MR diffusional kurtosis imaging has recently been proposed as a means of quantifying non-Gaussian water diffusion; in general, the regions of high fractional anisotropy are also characterized by increased mean kurtosis.

With regard to $\mathrm{PD}$, previous diffusion tensor imaging studies on affected patients have demonstrated a decrease in fractional anisotropy in SNc compared with the fractional anisotropy in SNc obtained in studies conducted on healthy control subjects. ${ }^{32,33}$ Patients with $\mathrm{PD}$ exhibit significantly higher $\mathrm{R}_{2}{ }^{*}$ values in the SNc, lower fractional anisotropy values in the SNc and thalamus, and higher mean diffusivity values in the thalamus than control subjects. ${ }^{1}$ However, the appearance of artifacts, which are more common in diffusion MR imaging sequences, depends on the local magnetic field. ${ }^{34}$ Inhomogeneities in the local magnetic field, such as those induced by iron deposition, lead to some artifacts in diffusion MR imaging sequences and have an effect on ADC. The sensitivity of the magnetic susceptibility changes because motion-probing gradients into echo-planar imaging are used in diffusion MR imaging sequences. Hence, it is possible that ADC is underestimated in the presence of iron depositions. Conversely, a 3D spoiled gradient-echo with a multiecho technique is more robust for evaluating nigrosome 1 than a diffusion MR imaging sequence in local magnetic field inhomogeneities. However, as mentioned earlier, the problem with the magic angle in the $\mathrm{B}_{0}$ direction affecting nigrosome 1 visualization became clear in this study.

There were some limitations to this study. The local field gradients by blood containing deoxyhemoglobin were paramagnetic. ${ }^{35}$ In the future, we will be able to evaluate the magnetic susceptibility of each tissue with more focus. Moreover, the effect of aging needs to be examined in detail. It has been reported that the mean diffusivity and fractional anisotropy are highly influenced by physiologic aging. ${ }^{36}$ Therefore, we will evaluate older subjects who are added to the study. The current MR imaging technique has made it possible to use appropriate parameters in this study (eg, the number or timing of combined echoes).

\section{CONCLUSIONS}

The visualization of nigrosome 1 is affected by the magic angle, thus causing asymmetric visualization. We observed that visualization was improved when the head was tilted to the right and left in the $\mathrm{B}_{0}$ direction. Furthermore, nigrosome 1 is affected by susceptibility. The cause of these problems in visualization is the anatomic slant structure of nigrosome 1; each anatomic slant structure individually has an effect on visualization.

Disclosures: Nobuyuki Arai-UNRELATED: Employment: Department of Radiology, Nagoya City University Hospital, Comments: The corresponding author had full access to all the data in the study and had final responsibility for the decision to submit for publication. Hirohito Kan-UNRELATED: Employment: Radiological and Medical Laboratory Sciences, Nagoya University Graduate School of Medicine, Comments: Assistant Professor. Masaki Ogawa - UNRELATED: Employment: Department of Radiology, Nagoya City University Graduate School of Medical Sciences. Yuto Uchida-UNRELATED: Employment: Department of Neurology, Nagoya City University Graduate School of Medical Sciences. Masahiro TakizawaUNRELATED: Employment. Hitachi Ltd., Comments: The sponsor had no control over the interpretation, writing, or publication of this work. Kazuyoshi OmoriUNRELATED: Employment: Hitachi Healthcare Ltd., Comments: The sponsor had no control over the interpretation, writing, or publication of this work. Tosiaki MiyatiUNRELATED: Employment: Division of Health Sciences, Graduate School of Medical Science, Kanazawa University, Comments: Professor. Harumasa Kasai-UNRELATED: Employment: Department of Radiology, Nagoya City University Hospital. Hiroshi Kunitomo-UNRELATED: Employment: Department of Radiology, Nagoya City University Hospital. Yuta Shibamoto-UNRELATED: Employment: Department of Radiology, Nagoya City University Graduate School of Medical Sciences, Comments: Professor.

\section{REFERENCES}

1. Péran $\mathrm{P}$, Cherubini A, Assogna F, et al. Magnetic resonance imaging markers of Parkinson's disease nigrostriatal signature. Brain 2010;133:3423-33 CrossRef Medline

2. Brass SD, Chen NK, Mulkern RV, et al. Magnetic resonance imaging of iron deposition in neurological disorders. Top Magn Reson Imaging 2006;17:31-40 CrossRef Medline

3. Berg D, Youdim MB. Role of iron in neurodegenerative disorders. Top Magn Reson Imaging 2006;17:5-17 CrossRef Medline

4. Griffiths $\mathrm{PD}$, Dobson $\mathrm{BR}$, Jones $\mathrm{GR}$, et al. Iron in the basal ganglia in Parkinson's disease: an in vitro study using extended x-ray absorption fine structure and cryo-electron microscopy. Brain 1999;122:667-73 CrossRef Medline

5. Graham JM, Paley MN, Grünewald RA, et al. Brain iron deposition in Parkinson's disease imaged using the PRIME magnetic resonance sequence. Brain 2000;123 Pt 12:2423-31 CrossRef Medline

6. Griffiths PD, Crossman AR. Distribution of iron in the basal ganglia and neocortex in postmortem tissue in Parkinson's disease and Alzheimer's disease. Dementia 1993;4:61-65 CrossRef Medline

7. Sedlacik J, Boelmans K, Lobel U, et al. Reversible, irreversible and effective transverse relaxation rates in normal aging brain at $3 \mathrm{~T}$. Neuroimage 2014;84:1032-41 CrossRef Medline

8. Aquino D, Bizzi A, Grisoli M, et al. Age-related iron deposition in the basal ganglia: quantitative analysis in healthy subjects. Radiology 2009;252:165-72 CrossRef Medline

9. Blazejewska AI, Schwarz ST, Pitiot A, et al. Visualization of nigrosome 1 and its loss in PD: pathoanatomic correlation and in vivo 7 T MRI. Neurology 2013;81:534-40 CrossRef Medline

10. Damier P, Hirsch EC, Agid Y, et al. The substantia nigra of the human brain, II: patterns of loss of dopamine-containing neurons in Parkinson's disease. Brain 1999;122:1437-48 CrossRef Medline

11. Cosottini M, Frosini D, Pesaresi I, et al. Comparison of 3T and 7T susceptibility-weighted angiography of the substantia nigra in diagnosing Parkinson disease. AJNR Am J Neuroradiol 2015;36:46166 CrossRef Medline

12. Damier P, Hirsch EC, Agid Y, et al. The substantia nigra of the human brain, I: nigrosomes and the nigral matrix, a compartmental organization based on calbindin $\mathrm{D}(28 \mathrm{~K})$ immunohistochemistry. Brain 1999;122:1421-36 CrossRef Medline

13. Schwarz ST, Afzal M, Morgan PS, et al. The 'swallow tail' appearance of the healthy nigrosome: a new accurate test of Parkinson's disease-a case-control and retrospective cross-sectional MRI study at 3T. PLoS One 2014;9:e93814 CrossRef Medline 
14. Wallis LI, Paley MN, Graham JM, et al. MRI assessment of basal ganglia iron deposition in Parkinson's disease. J Magn Reson Imaging 2008;28:1061-67 CrossRef Medline

15. Noh Y, Sung YH, Lee J, et al. Nigrosome 1 detection at 3T MRI for the diagnosis of early-stage idiopathic Parkinson disease: assessment of diagnostic accuracy and agreement on imaging asymmetry and clinical laterality. AJNR Am J Neuroradiol 2015;36:2010-06 CrossRef Medline

16. Nam Y, Gho SM, Kim DH, et al. Imaging of nigrosome $\mathbf{1}$ in substantia nigra at $3 \mathrm{~T}$ using multiecho susceptibility map-weighted imaging (SMWI). J Magn Reson Imaging 2017;46:528-36 CrossRef Medline

17. Bilgic B, Pfefferbaum A, Rohlfing T, et al. MRI estimates of brain iron concentration in normal aging using quantitative susceptibility mapping. Neuroimage 2012;59:2625-35 CrossRef Medline

18. Lee J, Nam Y, Choi JY, et al. Mechanisms of T2 * anisotropy and gradient echo myelin water imaging. NMR Biomed 2017;30 [Epub 2016 Apr 7] CrossRef Medline

19. Puwal S, Roth BJ, Basser PJ. Heterogeneous anisotropic magnetic susceptibility of the myelin-water layers causes local magnetic field perturbations in axons. NMR Biomed 2017;30 CrossRef Medline

20. Aggarwal M, Kageyama Y, Li X, et al. $\mathbf{B}_{0}$-orientation dependent magnetic susceptibility-induced white matter contrast in the human brainstem at 11.7T. Magn Reson Med 2016;75:2455-63 CrossRef Medline

21. Wu B, Li W, Guidon A, et al. Whole brain susceptibility mapping using compressed sensing. Magn Reson Med 2012;67:137-47 CrossRef Medline

22. Khabipova D, Wiaux Y, Gruetter R, et al. A modulated closed form solution for quantitative susceptibility mapping: a thorough evaluation and comparison to iterative methods based on edge prior knowledge. Neuroimage 2015;107:163-74 CrossRef Medline

23. Li W, Wang N, Yu F, et al. A method for estimating and removing streaking artifacts in quantitative susceptibility mapping. Neuroimage 2015;108:111-22 CrossRef Medline

24. Erickson SJ, Prost RW, Timins ME. The "magic angle" effect: background physics and clinical relevance. Radiology 1993;188:23-25 CrossRef Medline
25. Chappell KE, Robson MD, Stonebridge-Foster A, et al. Magic angle effects in MR neurography. AJNR Am J Neuroradiol 2004;25:431-40 Medline

26. Kan H, Arai N, Kasai H, et al. Quantitative susceptibility mapping using principles of echo shifting with a train of observations sequence on 1.5T MRI. Magn Reson Imaging 2017;42:37-42 CrossRef Medline

27. Wang Y, Liu T. Quantitative susceptibility mapping (QSM): decoding MRI data for a tissue magnetic biomarker. Magn Reson Med 2015;73:82-101 CrossRef Medline

28. Liu J, Liu T, de Rochefort $\mathrm{L}$, et al. Morphology enabled dipole inversion for quantitative susceptibility mapping using structural consistency between the magnitude image and the susceptibility map. Neuroimage 2012;59:2560-68 CrossRef Medline

29. de Rochefort L, Liu T, Kressler B, et al. Quantitative susceptibility map reconstruction from MR phase data using Bayesian regularization: validation and application to brain imaging. Magn Reson Med 2010;63:194-206 CrossRef Medline

30. Abe O, Aoki S, Hayashi N, et al. Normal aging in the central nervous system: quantitative MR diffusion-tensor analysis. Neurobiol Aging 2002;23:433-41 CrossRef Medline

31. Le Bihan D, Mangin JF, Poupon C, et al. Diffusion tensor imaging: concepts and applications. J Magn Reson Imaging 2001;13:534-46 CrossRef Medline

32. Vaillancourt DE, Spraker MB, Prodoehl J, et al. High-resolution diffusion tensor imaging in the substantia nigra of de novo Parkinson disease. Neurology 2009;72:1378-84 CrossRef Medline

33. Yoshikawa K, Nakata Y, Yamada K, et al. Early pathological changes in the parkinsonian brain demonstrated by diffusion tensor MRI. J Neurol Neurosurg Psychiatry 2004;75:481-84 CrossRef Medline

34. Fujiwara S, Uhrig L, Amadon A, et al. Quantification of iron in the non-human primate brain with diffusion-weighted magnetic resonance imaging. Neuroimage 2014;102:789-97 CrossRef Medline

35. Ogawa S, Menon RS, Tank DW, et al. Functional brain mapping by blood oxygenation level-dependent contrast magnetic resonance imaging: a comparison of signal characteristics with a biophysical model. Biophys J 1993;64:803-12 CrossRef Medline

36. Cherubini A, Peran P, Hagberg GE, et al. Characterization of white matter fiber bundles with $\mathrm{T}^{\star}$ relaxometry and diffusion tensor imaging. Magn Reson Med 2009;61:1066-72 CrossRef Medline 